\title{
ON THE SYNCHRONIZABILITY AND DETECTABILITY OF
}

\section{RANDOM PPM SEQUENCES}

$$
\text { N }
$$

\section{Technical Report III}

\section{0

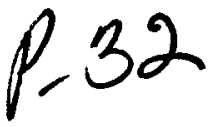

to

\author{
NASA \\ Goddard Space Flight Center \\ Greenbelt, Maryland
}

Grant Number NAG 5-778

\author{
Costas N. Georghiades \\ Principle Investigator \\ (acting for Shu Lin) \\ Department of Electrical Engineering Department \\ Texas A\&M Iniversity \\ rollege Station. Texas Tist?
}

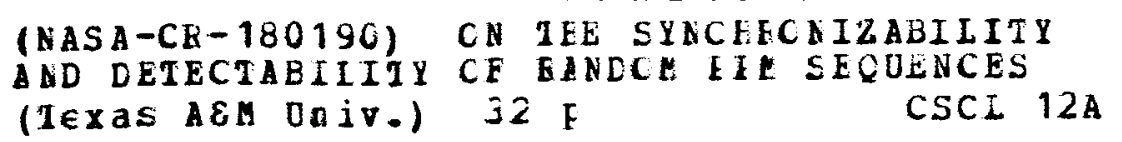

March 25. IMT 


\title{
ON THE SYNCHRONIZABILITY AND DETECTABILITY OF RANDOM PPM SEQUENCES
}

\author{
by \\ Costas N. Georghiades \\ Electrical Engineering Department \\ Texas A\&M University \\ College Station, TX 77843
}

\begin{abstract}
The problem of synchronization and detection of randon pulse-position-modulation (PPM) sequences is investigated under the assumption of perfert slot synchronization. Maximum-likelihood PPM symbol synchronization and receiver algorithms are derived that make decisions based both on soft as well as hard data: these algorithms are seen to be easily implementable. We derive bounds on the symbol error probability as well as the probability of false synchronization that indicate the existence of a rather severe performance floor, which can easily be the limiting factor in the overall system performance. The performance floor is inherent in the PPM format and random data and becomes more serious as the PPM alphabet size $Q$ is increased. A way to eliminate the performance floor is suggested by inserting "special" PPM symbols in the random data stream.
\end{abstract}




\section{INTRODUCTION}

Pulse-position-modulation is a modulation format known to be optimal in various ways for the direct-detection optical channel (see for example $[1,2,3]$ ). Under Q-ary PPM, information is contained in the position of a signal pulse in only one of $Q$ subintervals, known as slots, dividing the symbol interval.

When pulse-position-modulation is used in communication systems, the practice is to first achieve slot synchronization before attempting higher order synchronization and symbol decoding. Slot synchronization is usually obtained by using a tracking-loop as recently studied, for example, by Chen and Gardner [4] and Ling and Gagliardi [5;. Although optimal (in the maximum-likelihood as well as the mean-square-error sense) PPM slot and symbol synchronizers have also been recently derived $[6, \bar{T}]$, they have the disadvantage of being more complicated to implement compared to the tracking-loop synchronizers that are well understood and easy to implement. The difficulty in implementing the optimal syuchronizers stems from the need to record the exact arrival time of each detected photon. a task that may be difficult to achieve at high data rates and large signal intensities. A further reason that makes tracking-loops more desirable for slot synchronization is that they have been shown to result in receivers with symbol error probability performance within a fraction of a $\mathrm{dB}$ from the perfect slot synchronization case at reasonable signal levels [4]. We point out here that the authors in [4] are investigating the effects of slot synchronization errors only and thus assume that once slot synchronization is achieved, symbol synchronization is automatically obtained. This is equivalent to assuming that the only ambiguity in symbol synchronization is the ambiguity in slot synchronization, which in practice is not a valid assumption since slot synchronization does not imply symbol synchronization.

In this paper we investigate the problem of PPM symbol synchronization and decoding under the assumption of perfect slot synchronization. For Q-ary PPM, the existence of slot synchronization still leaves unresolved a Q-ary ambiguity as to the position of the PPM symbols. However. in contrast to the slot synchronization case where system error probability degrades gracefully with the slot synchronization error, the effect of non- 
perfect symbol synchronization is catastrophic. One can easily see that a sequence of $N$ random PPM symbols decoded under non-perfect symbol synchronization will result in all $N$ symbols being decoded erroneously, irrespective of the size of the error. This observation leads to the conclusion that the real bottleneck in system performance is due to the symbol synchronization subsystem which we investigate in the sequel.

In section II we investigate and characterize the synchronization properties of random PPM sequences. In section III, we derive ML symbol synchronization algorithms for the PPM, optical Poisson channel, both from soft as well as hard data. Here we also derive a bound on the synchronization probability, valid at high signal-to-noise levels. Section IV contains the derivation of optimal receivers that make sequence decisions in the absence of symbol synchronization. Also included in this section is a bound on the minimum achievable symbol error probability. Finally, we conclude with section V.

\section{SYNCHRONIZABILITY OF PPM SEQUENCES}

In this section we investigate the synchronization properties of random PPM sequences. Broadly speaking, these are properties associated with our ability to identify uniquely (or not) the location of PPM symbols within a sequence of such symbols when only slot boundaries are known. Before we proceed further, we introduce some definitions and terminology to establish a common ground and facilitate later analysis.

Definition 1: A binary sequence of $Q$ digits is said to satisfy the PPM constraint and, thus. be a Q-ary PPM symbol only if exactly one out of the $Q$ digits is a "one"; $Q$ will be referred to as the PPM alphabet size.

It is easily seen from the above definition that for a given sequence length $N$ there are $Q^{N}$ valid PPM sequences and that they can be thought of as a subset of the $2^{N Q}$ binary sequences of length $N Q$.

Definition 2: A sequence of binary digits is said to be a valid Q-ary PPM sequence if. starting from the first bit. crery consecutive subsequence in it of length $Q$ is a $Q$-ary PPM symbol. 
Definition 3: Consider a $Q$-ary PPM sequence $(N+1)$ symbols long $((N+1) Q$ slots $)$ and a sliding window of $N Q$ slots applied to the PPM sequence. For a given slot shift $j$, $j=0,1,2, \ldots,(Q-1)$, we will refer to the binary sequence within the window as a PPM binary subsequence at (slot shift) $j$. It is obvious from the definition that a PPM binary subsequence is not necessarily a PPM sequence, i.e., not all consecutive binary sequences of length $Q$ within the window are PPM symbols. Also obvious is the fact that if $j=0$, then the PPM binary subsequence is a PPM sequence that coincides with the first $N$ symbols of the original sequence. Similarly, for $j=Q$ the PPM binary subsequence is a PPM sequence that coincides with the last $N$ symbols in the original sequence. Notice that slot shift $j=Q$ does not need to be investigated in searching for symbol locations since it is equivalent $10 j=0$.

Given the above definitions, we are now ready to derive some results. As a first step in characterizing the synchronization properties of random PPM sequences, we are interested in the following problem.

Consider a Q-ary PPM sequence of length $\left(N^{\circ}+1\right)$ symbols, chosen at random from the set of $Q^{(N+1)}$ possible PPM sequences, and a given slot shift $j$. We are interested in the probability that exactly $K^{*}$ out of the $N$ symbols within the PPM binary subsequence at slot shift $j$ are Q-ary PPM symbols. The usefulness of this quantity should be apparent in the context of symbol synchronization when only slot synchronization is present. Leaving the details of the derivation for Appendix A, we can show that this probability, defined by $P\left(K^{-} ; j, Q, N\right)$, is given by

$$
P\left(K^{-} ; j . Q, N\right)=\left(\begin{array}{l}
N \\
K^{-}
\end{array}\right)\left\{(j / Q)^{(K+1)}(1-j / Q)^{\left(N-K^{-}\right)}+(1-j / Q)^{\left(K^{\prime}+1\right)}(j / Q)^{\left(N-K^{\prime}\right)}\right\} .
$$

It is obvious from the above expression that $P\left(K^{-} ; j, Q, N\right)=P\left(K^{-}, Q-j, Q, N\right)$, which implies that $P\left(K^{*}: j, Q, N\right)$ is symmetric with respect to forward and backward slot shifts from the correct slot for symbol synchronization. Another observation that can be readily made is that the above probability is a convex function of $j$. achieving a minimum at $j=Q / 2$ when $Q$ is even. as is usually the case. This implies that slots closer to the correct symbol synchronization slot have a higher probability of being erroneously chosen 
for symbol synchronization than slots further away.

Of special interest is the case when $K=N, P(N ; j, Q, N)$, i.e., the probability that the PPM binary subsequence at slot shift $j$ is a PPM sequence. Easily obtained from (1), it is

$$
P(N ; j, Q, N)=(j / Q)^{N+1}+(1-j / Q)^{N+1}
$$

Equation (2) implies that there is a nonzero probability of identifying symbol locations erroneously, even in the absence of noise. A bound on the probability of erroneous symbol synchronization is derived in the next section.

In passing. we note that $P\left(K^{-} ; j, Q, N\right)$ can be expressed as the ratio of the number of PPM secluences that, for a given $j$, result in exactly $K^{-}$PPM symbols within the PPM binary subsequence at $j$, to the total number of distinct PPM sequences of length $(N+1)$. Denoting the number of sequences resulting in $K$ matches by $D(K ; j, Q, N)$, we then have

$$
D\left(K_{;} j, Q . N\right)=Q^{(N+1)} \quad P\left(K^{-}: j, Q, N\right)
$$

We now turn our attention to the implications of (1) on symbol synchronization in the limit as the sequence length $N$ tends to infinity. It is readily seen that for any fixed $K, P(K ; j, Q, N) \rightarrow 0$ as $N \rightarrow \infty$, which implies that the fraction of sequences that result in exactly $K^{-}$matches diminishes with $N$. However, the more important quantity is the probability that the number of matches $K$ will exceed a given number $r$. Specifically, of interest is the smallest value of $r$ such that the probability of more than $r$ matches goes to zero as $Y \rightarrow x$. for all slot shifts $j=1,2$. $\cdots,(Q-1)$. Obviously, this minimum value of $r$ is a function of the PPM modulation format and the alphabet size $Q$ only; moreover, the smaller it is, the better the symbol synchronizer will be able to perform in the presence of noise for large $N$.

The following proposotion establishes the range of values of $r$ such that the probability of more than $r$ symbol matches tends to zero with $N$.

Proposition 1: Let $\operatorname{Pr}\left[\boldsymbol{H}^{-} \geq r ; j, Q, N\right]$ be the probability that the number of symbol matches $K^{-}$within a PPM binary subsequence at slot shift $j$ exceeds $r$ for a given $Q$ and 
$N$. Then, if $(r / N)>(1-1 / Q)$

$$
\lim _{N \rightarrow \infty} \operatorname{Pr}[K \geq r ; j, Q, N]=0
$$

for all slot shifts $j$. Conversely, for $(r / N)<(1-1 / Q)$, there is some slot shift $j$ such that

$$
\lim _{N \rightarrow \infty} \operatorname{Pr}\left[\hbar^{-} \geq r ; j, Q, N\right]=1
$$

Proof: We first prove (4). Using the Chernoff bound we have

$$
\operatorname{Pr}[K \geq r ; j, Q, N] \leq \exp (-s r) \phi(s ; j, Q, N) \quad s \geq 0
$$

where

$$
\begin{aligned}
Q(s ; j \cdot Q \cdot N) & =\sum_{n=0}^{N} \operatorname{cxp}(\operatorname{sm}) P(m ; j \cdot Q \cdot N) \\
& \left.\left.=(j / Q) !(j / Q) \epsilon^{*}+(1-j / Q)\right)^{N}+(1-j / Q)(1-j / Q) \epsilon^{*}+j / Q\right)^{N}
\end{aligned}
$$

is the characteristic function of the random variable $K$, easily obtained from (1). In order to guarantee that (4) holds for all $j$. it is enough to make sure that (4) holds for the $j$ that makes the right-hand side of (6) largest. From the easily established convexity of $o(s ; j, Q, N)$ and its symmetry around $j=Q / 2$, we conclude that the maximum occurs at one of the boundaries, say $j=1$. Letting $p=1 / Q$ we have

$$
\phi(s ; j, Q, N) \leq \phi(s ; 1, Q, N)=p\left[p \epsilon^{s}+(1-p)\right]^{N}+(1-p)\left[(1-p) \epsilon^{s}+p\right]^{N}
$$

Observing now that the second term in $(8)$ is the larger term we can further bound the right-hand side of $(8)$ by twice this term; combining with the bound in (6), we get

$$
\operatorname{Pr}\left[K^{-} \geq r: j . Q . N\right] \leq 2(1-p) \exp (-s r)\left[(1-p) \epsilon^{s}-p\right]^{N} . s \geq 0
$$

Further tightening the bound with respect to $s$, we finally obtain for $(r / N) \geq(1-1 / Q)$

$$
0 \leq \operatorname{Pr}\left[\begin{array}{r}
* \\
0
\end{array}, Q, N \leq 2(1-p) \exp \left\{-N\left[E(r / N \|(1-p))-h_{b}(r / N)\right]\right\}\right.
$$


In (10) $E[q \| h]$ and $h_{b}(x)$ are the cross entropy (inaccuracy) and binary entropy functions defined respectively by

$$
\begin{gathered}
E[q \| h]=-q \ln (h)-(1-q) \ln (1-h) \\
h_{b}(x)=-x \ln (x)-(1-x) \ln (1-x)
\end{gathered}
$$

for some probabilities $q, h$ and $x$. It is well known that the inaccuracy is always greater than or equal to the entropy (see for example [8]), which implies that the exponent in (10) is non-positive. Thus, in the region $(r / N)>(1-1 / Q)$ where the bound is valid, taking the limit as $N \rightarrow \infty$ yields (4).

We now turn our attention to the converse in (5). Since we only need to show convergence to unity for some $j$, we choose $j=(Q-1)$ (an educated choice). We start by deriving the following C'hernoff bound

$$
\left.\operatorname{Pr} \mid \Lambda^{-} \leq r ; j, Q, N\right] \leq \exp (-s r) \varphi(s ; j, Q, N), s \leq 0
$$

Following arguments paralleling those above we obtain for $j=(Q-1)$ and $(r / N) \leq$ $(1-1 / Q)$

$$
\operatorname{Pr}\left[K^{-}<r: j, Q, N\right] \leq 2(1-1 / Q) \exp \left\{-N\left[E(r / N \|(1-1 / Q))-h_{b}(r / N)\right]\right\}
$$

where we have used the fact that $\operatorname{Pr}\left[K^{-}<r ; j, Q, N j \leq \operatorname{Pr}\left[K^{-} \leq r ; j, Q, N\right]\right.$. The condition $(r / N) \leq(1-1 / Q)$ gives a range of values of $r$ for which the bound is valid and is derived from the condition $s \leq 0$. Taking limits as $N \rightarrow \infty$ in (14) we obtain $\operatorname{Pr}\left[K^{-}<r ; j, Q, N\right] \rightarrow 0$ when $(r / N)<(1-1 / Q)$, which in turn implies (5). This completes the proof of the proposition.

One of the implications of the theorem is that for large (theoretically infinite) $N$. the fraction of symbols within any PPM binary subsequence that are valid PPM symbols can be quaranteed to be less than $(1-1 / Q)$. In other words, the number of PPM symbol matches $K^{-}$for any slot shift $j=1,2, \cdots,(Q-1)$, satisfies, for large $N$

$$
K^{-}<(1-1 / Q) N
$$


which implies that the number of mismatched symbols, call it $d$, satisfies

$$
d=\left(N-K^{-}\right) \geq N / Q
$$

From (16) we can see that for large $N$, the minimum number of symbol mismatches for any slot shift $j$, call it $d_{\mathrm{min}}$, is

$$
d_{\min }=N / Q
$$

The quantity $d_{\min }$ can easily be paralleled to the minimum distance of a block code, only now $d_{\min }$ is the minimum average distance between random PPM sequences for any slot shift $j=1,2, \cdots,(Q-1)$ away from the correct synchronization slot. Clearly, the larger $d_{\text {min }}$ is. the better the symbol synchronizer will be able to identify symbol locations in the presence of noise. Another obvious observation from (17) is that $d_{\min }$ is monotonically decreasing with the PPM alphabet size $Q$, which in turn implies that synchronization performance deteriorates as $Q$ is increased. This observation was made also in [4] and [7] for the slot and PPM symbol synchronization problems respectively. Later in this paper we relate the minimum distance $d_{\min }$ for random PPM sequences to the smallest achievable synchronization error probability.

In concluding this section, we note that the minimum average distance $d_{\text {min }}$ derived above is for random PPM sequences, which indicates the existence of sequences with larger $d_{\min }$. The problem of designing such sequences is briefly investigated in the next section.

We next turn our attention to the problem of deriving optimal PPM symbol synchronization when slot synchronization is present.

\section{ML SYNCHRONIZATION}

Our application area here is the direct-detection optical Poisson channel. For this channel, it can be easily established that when only slot synchronization is present, the sufficient statistic is the number of photons (events) observed in each slot interval of $T^{\prime}$ seconds. For the Poisson direct-detection channel. the number of counts observed in distinct slot intervals are independent. Poisson random variables with intensity $\left(\lambda_{s}+\lambda_{n}\right)$ if a signal pulse is present in the corresponding slot and $\lambda_{n}$ otherwise: $\lambda_{s}$ and $\lambda_{n}$ are known 
as the signal and noise intensities respectively. We derive synchronization algorithms from two kinds of observations, defined in the sequel.

\section{a) Soft-data observations:}

In deriving the $M L$ synchronization algorithm here, we assume that the receiver has available an observation vector $\mathbf{R}=\left(K_{1}, K_{2}, \cdots, \hbar_{Q}^{-}, \cdots, \hbar_{N Q}\right)$ with elements being the number of counts in $N Q$ observed, consecutive slots. We will refer to the data in $\mathbf{R}$ as soft-data. Within this observation window there are $(N+1)$ symbols, two of them at the boundaries partially contained. The problem is to estimate the location of symbols within the observation interval.

Denoting by $m$ the variable associated with the start of a symbol within the first $Q$ slots in the observation interval, a $M L$ synchronizer implements the following

$$
\max _{0 \leq m \leq i Q-1)} \sum_{\mathbf{d}} \operatorname{Pr}(\mathbf{R} ; m . \mathbf{d})
$$

where $\mathbf{d}=\left(d_{1}, d_{2}, \cdots, d_{Q}, \cdots, d_{N Q}\right)$ is the random modulation vector of intensities within the observation interval. In writing (18), we assume that all modulation vectors are equiprobable. Following the general approach described in $[6,7]$ and making the assumption that the first partially contained symbol within the observation interval is a continuation of the last partially contained symbol, we obtain as the likelihood statistic

$$
L_{s}(m)=\sum_{i=1}^{N} \ln \left\{\sum_{j=1}^{Q} \exp \left[\ln (X) K_{(i-1) Q+j+m}\right\}\right\} \text {. }
$$

In (19). $I=\left(1-\lambda s / \lambda_{n}\right)$ and $K_{i}$ is the number of counts observed in the $i-$ th slot in the observation interval. The indexes in (19) are interpreted modulo $Q N$ to account for our approximation above. We note here that this approximation was not necessary in deriving the $M L$ synchronizer but was made to reduce complexity at no practical performance loss for values of of $N$ greater than four $[6,7$.

A further approximation to (19) can be obtained by using only the largest term in the sum over $j$ to yield

$$
L^{\prime}(m)=\sum_{i=1}^{N} h_{(i-1) Q-j_{i}+m}
$$


We will refer to $(20)$ in the sequel as the max-rule. In $(20), j_{i}$ is the value of $j, 1 \leq j \leq Q$, that, for a given $i$ and $m$ maximizes $K_{(i-1) Q+j+m}$.

Computer simulation results comparing the probability of correct synchronization, $P_{c s}$, for the synchronizers in (19) and (20) are reported in Figures 1,2 and 3 for different values of $Q$ and $N$. These results indicate that the much simpler synchronizer in (20) performs practically as well as the significantly more complicated one in (19). Also evident from the graphs is that for the same signal energy per slot $\lambda_{s} T^{\prime}$, synchronization performance degrades with $Q$ and improves with $N$, as predicted in the previous section. Finally. it is clear from the figures that a performance floor exists which is practically in effect for signal levels of about $\lambda_{s} T^{\prime}=10.0$. This error-floor is investigated later in this section.

We now turn our attention to deriving synchronization algorithms when the receiver observations consist of hard-data. obtained from the soft-data vector $\mathbf{R}$ by making hard decisions in each slot interval.

\section{b) Hard-data observations}

Here we assume that the receiver has available the binary vector of observations $\mathbf{X}=$ $\left(x_{1}, x_{2}, \cdots, x_{Q}, \cdots, x_{N Q}\right)$ obtained by making hard-decisions on the vector $\mathbf{R}$. By this we mean that the receiver looks at $K_{i}^{-}, i=1,2, \cdots, N Q$, and decides that $x_{i}=1$ or $x_{i}=0$ according to whether a signal pulse is detected (a "one") or not (a "zero") respectively; i.e..

$$
\frac{\operatorname{Pr}\left[\text { "one" } / R_{i}=K_{i}\right]}{\operatorname{Pr}\left[\text { "zero" } / R_{i}=K_{i}\right]} \underset{x_{i}=u}{>} \gtrless^{x_{i}=1} 1 .
$$

In (21). $R_{\text {; }}$ is the (Poisson) random variable associateed with the number of counts in the $i$-th slot and $K_{i}$ are the observed counts. The above test can easily be shown to reduce to

$$
\begin{array}{rl}
h_{i} & > \\
x_{i}=u & i
\end{array}
$$

where the optimal threshold $\gamma$ is given by

$$
\eta=\frac{\lambda, T^{\prime}-\ln (Q-1)}{\ln (1-\lambda s / \lambda n)}
$$


It is obvious that further processing on the vector $\mathbf{X}$ to derive synchronization and perform decoding is much easier to implement compared to the soft-data case. Another advantage of algorithms derived from hard-data is that their structure does not depend on the complete statistics of the channel. The above reasons were partly why hard-data was employed in $[9$.

Before we proceed with the derivation of the ML synchronization algorithm, we introduce the following quantities:

$$
\begin{aligned}
& \operatorname{Pr}\left[X_{i}=0 / d_{i}=0\right]=\operatorname{Pr}\left[K_{i}<\gamma / d_{i}=0\right] \triangleq P_{00} \\
& \operatorname{Pr}\left[I_{i}=1 / d_{i}=1\right]=\operatorname{Pr}\left[K_{i} \geq \gamma / d_{i}=1\right] \triangleq P_{11} .
\end{aligned}
$$

Then

$$
\begin{gathered}
\operatorname{Pr}\left[X_{i=1} / d_{i}=0\right]=\left(1-P_{00}\right) \\
\operatorname{Pr} X_{i}=0 / d_{i}=1=\left(1-P_{11}\right)
\end{gathered}
$$

The above probabilities can be easily precomputed given $T^{\prime}, Q$ and the signal and noise intensities $\lambda_{s}$ and $\lambda_{n}$ respectively. Inder the assumption of equiprobable PPM symbols. the ML synchronization rule maximizes over $0 \leq m \leq(Q-1)$ the following statistic

$$
\begin{aligned}
& L_{1}(m)=\sum_{\mathbf{d}} \operatorname{Pr}[\mathbf{X} / m, \mathbf{d}] \\
& =\prod_{i=0}^{N-1} \sum_{k=1}^{Q} \prod_{\substack{j=1 \\
j \neq k}}^{Q} \operatorname{Pr}\left[X_{i Q+j+m}=x_{i Q+j+m} / m, d_{i Q+j+m}=0\right] \\
& \times \operatorname{Pr}\left[X_{i Q+k+m}=x_{i Q+k+m} / m, d_{i Q+k+m}=1\right] \\
& =\prod_{i=0}^{N-1} \prod_{j=1}^{Q} \operatorname{Pr}\left[X_{i Q+j+m}=x_{i Q+j+m} / m, d_{i Q+j+m}=0\right. \\
& \times \sum_{k=1}^{Q} \frac{\operatorname{Pr}\left[X_{1} Q+k+m=x_{i} Q+k+m i m, d_{i} Q+k+m=1\right]}{\operatorname{Pr}\left[X_{1} Q-k+m=x_{i} Q+k+m / m, d_{i} Q+k+m=0\right]}
\end{aligned}
$$

The second equality above is a result of the independence between choice of PPM symbols. which breaks the expectation over sequences to expectations over individual symbols. 
The third equality should be self evident. Observing now that the product over all $j$ 's and $i$ 's of $\operatorname{Pr}\left[X_{i Q+j+m}=x_{i Q+j+m} / m, d_{i Q+j+m}=0\right]$ is not a function of $m$, we can equivalently maximize,

$$
L_{2}(m)=\sum_{i=0}^{N-1} \ln \left[\sum_{k=1}^{Q} l_{i Q+k+m}^{\prime}\right],
$$

where

$$
l_{i Q+k+m}^{\prime}=\frac{\operatorname{Pr}\left[X_{i Q+k+m}=x_{i Q+k+m} / m, d_{i Q+k+m}=1\right]}{\operatorname{Pr}\left[X_{i Q+k+m}=x_{i Q+k+m} / m, d_{i Q+k+m}=0\right]} .
$$

In deriving (27), we have dropped terms not dependent on $m$ and taken the logarithm of the resulting expression. Further noting that by multiplying $l_{i Q+k+m}^{\prime}$ by any constant does not affect the maximization. we finally obtain

$$
L_{h}(m)=\sum_{i=0}^{N-1} \ln \left[\sum_{k=1}^{Q} l_{i Q+k+m}\right]
$$

where

$$
l_{i Q+k+m}=\frac{P_{11}}{\left(1-P_{00}\right)} l_{i Q+k+m}^{\prime} .
$$

It is clear that $l_{i Q+k+m}$ takes one of two values according to

$$
l_{i}= \begin{cases}1, & \text { if } x_{i}=1 \\ C, & \text { if } x_{i}=0\end{cases}
$$

where

$$
C=\frac{\left(1-P_{11}\right)\left(1-P_{00}\right)}{P_{11} P_{00}} .
$$

Since for a practical system $P_{11} \geq\left(1-P_{11}\right)$ and $P_{00} \geq\left(1-P_{00}\right)$, it is $0 \leq C \leq 1$. For a good system $\left(P_{11}>>\left(1-P_{11}\right)\right.$ and $\left.P_{00}>>\left(1-P_{00}\right)\right) C$ will be very close to zero and exactly zero when either $P_{00}$ or $P_{11}$ is unity. In this case. the optimal synchronizer from hard data reduces to

$$
L_{h}(m)=\sum_{i=0}^{N-1} \ln \left[\sum_{k=1}^{Q} x_{i Q+k+m}\right] .
$$

A situation when $P_{00}=1$ arises, for example. when $\lambda_{n}=0$ resulting in an erasure channel. It is then seen that the optimal synchronization rules for a perfect channel where both "zeros" and "ones" are decoded correctly with probability one is the same as that 
for the erasure channel where only "zeros" are detected correctly all the time. This of course does not imply that the performance of the synchronizers for the two channels are identical. From the information theory point of view, for both the perfect and the erasure channels, it is the presence of a pulse that carries all synchronization information, utilized fully by (33).

In general, for channels with small noise intensities $\lambda_{n}$ and relatively large signal intensities $\lambda_{s},(33)$ can be used as an approximation to (29) to further reduce complexity.

Having derived optimal synchronization under the assumptions of both soft as well as hard-data. we now turn our attention to the investigation of the ultimate performance achievable by such synchronizers.

c) A bound on svnchronization probability

Our interest here is deriving an upper bound to the probability of correct synchronization. $P_{\ldots}$. It is clear that the synchronization probability is bounded from above by the probability that one or more PPM binary subsequences are PPM sequences, in which case a random selection must be made. Denoting this random sequence limited probability by $P_{\text {rsl }}$, we have

$$
P_{c s} \leq P_{r s l}=Q^{-(N+1)} \sum_{k=0}^{Q-1} \frac{D_{k}}{k+1}
$$

where $D_{k}$ is the number of PPM sequences resulting in exactly $k$ slot shifts for which PPM binary subsequences are valid PPM sequences. The probability of $k$ such matches, $P_{m}(k)$, is

$$
P_{m}(k)=D_{k} \cdot Q^{-(N+1)}
$$

Leaving the details for Appendix B. we can derive the following expressions

$$
\begin{gathered}
D_{0}=Q^{(N+1)}-2(Q-1)^{(N-1)}-(Q-2)^{(N+1)} \\
P_{m}(0)=1-2(1-1: Q)^{(N-1)}-(1-2 / Q)^{(N+1)} \\
D_{1}= \begin{cases}2, & Q=2 \\
2\left[(Q-1)^{(N+1)}-(Q-2)^{(N+1)}-1\right], & Q>2\end{cases}
\end{gathered}
$$




$$
P_{m}(1)= \begin{cases}2^{-N}, & Q=2 \\ 2\left[(1-1 / Q)^{(N+1)}-(1-2 / Q)^{(N+1)}-(1 / Q)^{(N+1)}\right], & Q>2\end{cases}
$$

and

$$
\begin{gathered}
D_{Q-1}=Q \\
P_{m}(Q-1)=Q^{-N} .
\end{gathered}
$$

The derivation of the above equations was obtained by repeated use of the following observation.

Proposition 2: For a given PPM sequence, in order for the PPM binary subsequence at $j$ to be a valid PPM sequence it is necessary and sufficient that either all pulses (pulsed slots) are before the $j$-th slot or all after the $j$-th slot.

The proof of the above proposition is easily seen by construction.

We are now ready to derive a bound on the random sequence limited probability $P_{r s l}$, and by extension to the probability of correct synchronization $P_{c s}$.

Proposition 3: The following bound on the random sequence limited probability $P_{r s l}$ holds for all $Q$ and $N$

$$
P_{r s l} \leq 1-(1-1 / Q)^{(N+1)}+\frac{1}{3}(1-2 / Q)^{(N+1)}
$$

with equality for $Q=2$ and $Q=3$.

Proof: The following inequality is obvious

$$
P_{r s l} \leq P_{m}(0)+\frac{1}{2} P_{m}(1)+\frac{1}{Q} P_{m}(Q-1)+\frac{1}{3} \sum_{k=2}^{Q-2} P_{m}(k)
$$

where equality is when $Q$ equals two or three. ling the fact that

$$
\sum_{k=2}^{Q-2} P_{m}(k)=1-\left[P_{m}(0)+P_{m}(1)-P_{m}(Q-1)\right]
$$

and the expressions for the various probabilities in equations (37), (39) and (41) we obtain the required bound. 
We note that the bound in (42) will be tight for all values of $Q$ when $N$ is sufficiently large to make the probability of two or more slot shifts for which PPM binary subsequences are PPM sequences negligibly small. This fact is verified in Table 1 , where simulation results are compared to our bound.

A lower bound on the probability of erroneous synchronization $P_{e s}=\left(1-P_{c s}\right)$ is

$$
P_{e s} \geq(1-1 / Q)^{(N+1)}-\frac{1}{3}(1-2 / Q)^{(N+1)}
$$

which is achieved at high signal-to-noise levels and sufficiently large $N$. It is clear from (45) that the error-floor rises with increasing $Q$ and decreasing $N$ as predicted by the decrease in $d_{\min }$ in equation $(17)$. Let us now show that the distance $d_{\min }$ enters the bound on erroneous synchronization probability in a natural way as $N \rightarrow x$. For a given $d_{\min }$, we have $Q=N / d_{\min }$. Then

$$
\begin{aligned}
& \lim _{\lambda \rightarrow \infty} P_{e s} \geq \lim _{V \rightarrow \infty}\left\{\left(1-\frac{d_{\min }}{\Lambda}\right)^{N-1}-\frac{1}{3}\left(1-\frac{2 d_{\min }}{N}\right)^{N+1}\right\} \\
& =\epsilon^{-d_{\min }}-\frac{1}{3} \epsilon^{-2 d_{\min }} .
\end{aligned}
$$

Since the second term above is at least an order of magnitude smaller than the first for reasonably large $d_{\min }$ ( say $d_{\min } \geq 3$ ), we obtain, for very large (theoretically infinite $N$ ) and reasonably large $d_{\min }$

$$
P_{e s} \geq \epsilon^{-d_{\min }}
$$

Equation $(47)$ indicates an exponential decrease in the error-floor with increasing $d_{\min }$.

As an example of how the bound in (4.5) can be used in a system design we derive next the minimum number of slots that need to be processed in order to quarantee a given performance. The number of slots observed, $U^{-}=Q(N+1)$, is a measure of the receiver complexity and is an important parameter in practical implementation. We show that the following proposition is true.

Proposition 4: The smallest number of slots $W_{\min }$ that need to be processed to guarantee an error-floor not more than some $P_{e f}$ is

$$
W_{\min }=-2 \log _{2}\left(P_{\epsilon f}\right)
$$


and is achieved with binary PPM.

Proof: We have $W=Q(N+1) \geq 2(N+1)$ with equality iff $Q=2$. Further, we have

$$
(1-1 / Q)^{(N+1)}-\frac{1}{3}(1-2 / Q)^{(N+1)}=P_{e f}
$$

which implies $(N+1) \geq-\log _{2}\left(P_{\text {ef }}\right)$ with equality iff $Q=2$. Combining the two inequalities above, we get

$$
W \geq-2 \log _{2}\left(P_{e f}\right)
$$

with equality iff $Q=2$. This proves the proposition.

We end this subsection by noting that the bound in (42) is valid also for the results reported in 12 ? where PPM symbol synchronization is derived in the absence of slot synchronization. Next, we investigate ways to remove the error floor predicted above.

\section{d) Sequence Design}

We start with a definition.

Definition 4: A PPM sequence will be said to be synchronizable if no PPM binary subsequence in it is a valid PPM sequence. Equivalently, a PPM sequence is synchronizable if each PPM binary subsequence of it contains at least one symbol that is not a PPM symbol.

It is clear from the above definition that symbol locations within synchronizable PPM sequences can be uniquely identified in the absence of noise. As we derived earlier, for a given $Q$ and $N$ there are $D_{0}$ synchronizable PPM sequences as given by equation (36).

We are now ready to prove the following propositions.

Proposition 5: When a synchronizable PPM sequence is inserted in another PPM sequence, the resulting longer sequence is synchronizable.

Proof: By the definition of a PPM sequence all symbols within it must be PPM symbols. However. for any slot shift $j=1.2 . \cdots,(Q-1)$. there is at least one symbol in the inserted synchronizable sequence that is not a PPM symbol (by definition). Since the inserted sequence is a part of the longer sequence. this implies that at least one symbol in the longer sequence is not a PPM symbol for all $j=1,2, \cdots,(Q-1)$. This implies by 
definition 4 that the longer sequence is synchronizable, which completes the proof.

Proposition 6: A $Q$-ary sequence is synchronizable if and only if it contains both symbols 1 and $Q$; symbol 1 has the pulse in the first slot and symbol $Q$ has the pulse in the $Q$-th slot.

Proof: a) We first prove the forward statement. If both symbols 1 and $Q$ are in the sequence. then there is no slot shift $j=1,2, \cdots,(Q-1)$ for which all pulses are before or after $j$. Thus, by proposition 2 no PPM binary subsequence is a PPM sequence and, thus, by definition 4 the sequence is synchronizable.

b) If not both symbols 1 and $Q$ are in the sequence, we distinguish three possibilities: only symbol 1 or only symbol $Q$ is present or neither is present. If symbol 1 only is present, then the statement "all pulses are before slot $(Q-1)$ " is true which by proposition 2 it implies the sequence is not synchronizable. Similarly, if symbol $Q$ only is present, then the statement "all pulses are after slot 1 " is true which implies again that the sequence is not synchronizable. Finally, if neither symbol is present, both of the above statements in quotation marks are true, which again implies the sequence is not synchronizable. This completes the proof.

To facilitate reference in the sequel, we will refer to the pair of symbols 1 and $Q$ as the synchronizable pair.

It is clear from the above proposition that $D_{0}$ in equation (36) gives the number of sequences from the set of $Q^{(N+1)}$ possible sequences that contain at least one synchronizable pair. Obvious from the proof of the proposition, also, is the fact that it doesn't matter where the two symbols are located within a sequence to make it synchronizable. Finally. we observe that the more synchronizable pairs a sequence contains, the better its synchronization properties in the presence of noise.

One way of removing the error-floor predicted by (42) is to periodically insert in the random data stream a syuchronizable pair. If. for example, a synchronizable pair is inserted every $L$ PPM symbols, all PPM sequences of length greater or equal to $L$ will be synchronizable according to proposition 5 . The efficiency $E$ of such a scheme as measured 
by the number of information symbols per transmitted symbol will be

$$
E=\frac{L}{L+2}=1-2 / L
$$

which approaches unity as $L$ is increased. It is clear, however, that the smaller $L$ is, the better chances for correct synchronization in the presence of noise will be. In cases where a special synchronization pattern is inserted in the data stream to facilitate frame synchronization (see for example $[10,11,12]$ ), the extra reduction in efficiency to improve symbol synchronization can be avoided by choosing a pattern with as many synchronizable pairs as possible. Such a pattern will aid in both symbol as well as frame synchronization when they are obtained separately.

In the next section, we investigate the problem of deciding what the symbols within an observation interval are as opposed to where they are which we analyzed above. We do this under the assumption of slot synchronization only.

\section{DETECTABILITY OF PPM SEQUENCES}

\section{a) ML Receivers:}

In this section we turn our attention to the problem of PPM sequence estimation when only slot locations are exactly known. Our observations consist of the vector $\mathbf{R}=$ $\left(K_{1}^{-}, K_{2}^{-}, \cdots, K_{Q}^{-}, \cdots, \hbar_{N Q}^{-}\right)$of slot counts.

Ender the assumption of equiprobable sequences, an optimal receiver is a ML receiver that chooses as its sequence estimate the sequence $d$ that maximizes

$$
p(\mathbf{R} / \mathbf{d})=\sum_{m=0}^{Q-1} p(\mathbf{R} / \mathbf{d}, m) \operatorname{Pr}(m)
$$

In (50). $\operatorname{Pr}(m)$ is the a priori probability that the first PPM symbol in the interval $\left[0 . Q T^{\prime}\right.$, starts at time $m T^{\prime}$. Assuming that no a priori knowledge exists, then $\operatorname{Pr}(m)=1 / Q$ for all m. I sing the fact that the elements of $\mathbf{R}$ are conditionally independent. Poisson random variables and dropping terms not dependent on the modulation sequence. we obtain as our 
optimal receiver

$$
\max _{\mathbf{d} \in \Delta} \ell(\mathbf{d})=\sum_{m=0}^{Q-1} \exp \left[\ln (X) \sum_{i=1}^{N} K_{J_{i}(\mathbf{d})+m}\right] .
$$

In deriving (51) we have assumed that the first symbol in the observation interval is a continuation of the last, partially contained symbol. As for the synchronization case, this approximation reduces substantially the complexity of the receiver at no practical performance loss for $N$ greater than about four [11]. To accommodate this approximation, indexes in (51) are interpreted modulo $Q N$. The set $\Delta$ is the set of all possible distinct Q-ary PPM sequences of length $N$ and $J_{i}(\mathbf{d}), i=1,2, \cdots, N$, is a set of indexes indicating the slots within sequence $d$ that contain the pulses. For example, the $Q=4$ PPM sequence consisting of the $N=5$ symbols $\{1,3,3.4 .2\}$ can be described equivalently by $\left\{J_{i}(\mathbf{d})\right\} \underset{i=1}{\stackrel{N}{N}}=\{1,7,11,16,22\}$. Finally $X=\left(1+\lambda_{s} / \lambda_{n}\right)$ is as defined earlier.

Although each computation of $(51)$ is relatively easy to perform, the complexity of the receiver is still overwhelming since $Q^{N}$ statistic need to be evaluated before a decision is made. However, following arguments similar to those in 11 . we can show that only $Q$ of the $Q^{N}$ sequences are most likely to have been sent. Denoting the set of $\mathrm{Q}$ candidate sequences by $\Delta^{*}$, the receiver in $(51)$ becomes (with no loss in performance)

$$
\max _{\mathbf{d} \in \Delta^{*}} \ell(\mathbf{d})
$$

The set of $Q$ candidate sequences $\Delta^{*}$ is obtain in the following way:

Step 1: Group the observation vector into $Q$ consecutive slots per symbol and decode it into a sequence of $N$ PPM symbols. Store the decoded sequence as a possible candidate. Note that decoding the observation vector into PPM symbols is done by choosing the largest number of counts in each group of $Q$ slots, which is the optimal strategy when synchronization is present.

Step 2: Cyclically shift the observation vector by one slot to the left and go back to step 1. Repeat until the observation vector is cyclically shifted by $(Q-1)$ slots.

The result of the above procedure is $Q$ candidate sequences which are then used to evaluate $\ell(\mathbf{d})$ and make a final decision by choosing the largest. The idea behind the 
tremendous reduction in the number of evaluations of $\ell(d)$ is simple: we know that symbols start at one of the times $m T^{\prime}, m=0,1 \cdots,(Q-1)$ corresponding to the start of the first $Q$ slots in the observation interval. Thus, by decoding the observation vector for each possible $m=0,1, \cdots,(Q-1)$, we are assured that one of the $Q$ decoded sequences is the one we would have obtained have we had perfect synchronization. This implies that limiting our search in the set $\Delta^{*}$ does not increase our error probability, since the best we can hope to achieve is the performance of a perfectly synchronized receiver.

To illustrate the above approach, we take a simple example.

Example 1: Let $Q=3, N=4$ and the observation vector $\mathbf{R}=(0,4,2,1,5,3,2,3,1,6,3,5$, Decoding this vector into a PPM sequence. we obtain $d_{0}=(2,5,8,10)$. Cyclically shifting $\mathbf{R}$ by one slot to the left and decoding we obtain $\mathbf{d}_{1}=(1,4,9,11)$. Finally shifting by another slot we get $\mathbf{d}_{2}=(3,4,8,10)$. Evaluating $(52)$ using $\ln (X)=1.3$ we get $\ell\left(\mathbf{d}_{0}\right)=$ $1.462 \times 10^{10} \cdot \ell\left(\mathbf{d}_{1}\right)=1.957 \times 10^{11}$ and $\ell\left(\mathbf{d}_{2}\right)=5.335 \times 10^{10}$ and thus the decision is $\mathbf{d}=\mathbf{d}_{1}=(1,4,9,11)$. In a practical implementation of $(52)$ only the first $(N-1)$ symbols in the decoded sequence will be retained as valid symbols since the last one is actually the concatenation of two partially complete symbols and is likely to be in error. For our example above only the subsequence $(1.4,9)$ will be retained. By properly allowing overlap between consecutive observation intervals, all symbols are effectively decoded.

Approximations to (51) can be derived that further reduce complexity. An obvious one is to maximize with respect to $\mathbf{d} \in \Delta^{*}$ not the sum over all $m$ but only the largest term in the sum. i.e.

$$
\max _{\mathbf{d} \in \lambda^{*}} \sum_{i=1}^{N} K_{J_{i}(\mathbf{d})+m^{*} \cdot(\mathbf{d})} \text {. }
$$

In $(5.3) m^{*}(\mathbf{d})$ is the value of $m=0.1, \cdots,(Q-1)$ that maximizes the inside sum in $(51)$ - for a given $\mathbf{d}$. What we have in effect is a joint estimation of the modulation sequence and synchronization. i.e. (5.3) is equivalent to

$$
\max _{\mathrm{d} \in \Delta^{*},} \sum_{m \in[0,(Q-1)]}^{N} \sum_{i=1}^{N} \hbar_{J_{i}(\mathbf{d})+m}
$$

We next turn our attention to the performance achievable by the above receivers. 


\section{b) A bound on error probability}

As with the synchronization problem, here we are interested in finding a bound on the ultimate performance that can be achieved by the receivers described above.

Our starting point is the observation that when a receiver detects a sequence at the wrong location, then the sequence is detected erroneously. This implies that the probability of sequence detection $P_{s d}$ is bounded by the probability of correct synchronization $P_{c s}$, i.e., $P_{s d} \leq P_{c s}$, which in turn yields

$$
P_{s d} \leq P_{r s l} \leq 1-(1-1 / Q)^{(N+1)}+\frac{1}{3}(1-2 / Q)^{(N+1)}
$$

We observe that the PPM format imposes a severe error-floor on the sequence detection probability when symbol synchronization is absent.

We now turn our attention to the symbol detection probability $P_{s y d}$ and prove the following proposition.

Proposition 7: The symbol detection probability is bounded by

$$
P_{s y d} \leq 1-(1-1 / Q)^{(N+1)}+\frac{1}{3}(1-2 / Q)^{(N+1)}
$$

Proof: Letting $\bar{P}_{s y d}$ be the symbol detection probability in the absence of any channel noise. we have $P_{s y d} \leq \bar{P}_{s y d}$. We further observe that, in the absence of noise. when a sequence error is made (due to wrong synchronization), all the symbols in a sequence are received incorrectly; otherwise, all symbols are received correctly. From this observation we infer that $\bar{P}_{s y d}$ equals the probability of sequence detection in the absence of noise, which in turn equals $P_{r s l}$.i.e., $\bar{P}_{s y d}=P_{r s l}$. We thus have $P_{s y d} \leq P_{r s l}$ which with equation (42) imply (56). This completes the proof.

The symbol error probability floor predicted $b y(56)$ in the absence of symbol synchronization can be very severe, especially for large values of $Q$. As an example. $Q=256$ is an alphabet size considered for some applications: if an error-probability floor of at most $10^{-3}$ is required. a simple calculation shows that at least 435,200 slots need to be processed $(N=1700)$ in order to satisfy the performance requirements. 


\section{CONCLUSIONS}

We have considered the problem of synchronization and detection of random PPM symbols in the presence of only slot synchronization. In characterizing the synchronization properties of PPM symbols imbedded in long random PPM sequences, we introduced the minimum distance $d_{\min }$. From our analysis we concluded that synchronization performance improves with the length of the observed sequence and degrades with increasing PPM alphabet size.

In section III we derived optimal and suboptimal symbol synchronizers and a lower bound to the synchronization error probability; the error floor was seen to be due to the PPM modulation format. A way to remove the error-floor was suggested which consists of inserting periodically in the random data stream a pair of "special" symbols. The insertion of these symbols may be necessary to remove the error-floor, especially for large values of $Q$ where it is most severe. Maximum-likelihood receivers that make sequence decisions in the presence of slot synchronization only are derived in section IV as well as bounds to the sequence and symbol detection probabilities. We observed here that the symbol detection probability is bounded by the probability of correct synchronization.

Our conclusion is that for channels where the mechanisms that can cause random slot shifts are such that the phase in a given observation interval cannot be reliably predicted from previous observation intervals, the symbol error-floor is severe, especially for large $Q$. In these cases, some signal design to eliminate the error-floor is necessary.

\section{REFERENCES}

1. J.R. Pierce, "Optical channels: Practical Limits with Photon Counting," IEEE Transactions on Communication, Vol. Com.-26, No. 12, pp. 1819-1821, December 1978.

2. D.L. Snyder and I.B. Rhodes, "Some Implications of the Cutoff-Rate C'riterion for Coded Direct-Detection Communication Systems." IEEE Transactions on Information Theory. Vol. IT-26, pp. 327-338, May 1980.

3. D.L. Snyder and C.N. Georghiades, "Design of Coding and Modulation for a Power 
Efficient Use of a Bandlimited Optical Channel,"IEEE Transactions on Communications, Vol. COM-31, No. 4, pp. 560-565, A pril 1983.

4. C.C. Chen and C.S. Gardner. "Performance of PLL Synchronized Optical PPM Communication Systems." IEEE Transactions on Communications, Vol. COM-34, No. 10, October 1986 .

5. G. Ling and R.M. Gagliardi, "Slot Synchronization in Optical PPM Communications," IEEE Transactions on Communications, Vol. COM-34, No. 12, December 1986.

6. C.N. Georghiades, "Maximum-Likelihood Symbol Synchronization for the DirectDetection Optical Onoff-heying Channel," to appear in the IEEE Transactions on Communications May 1987.

7. C.N. Georghiades, "Optimum Joint Slot and Symbol Synchronization for the Optical PPM Channel." to appear in the IEEE Transactions on Communications May 1987.

४. R. Gallager. Information Theory and Reliable Communication. Wiley \& Sons 1968.

9. J. Lesh. J. Katz, H. Tan and D. Zwillinger, "2.5 Bit/Detected Photon Demonstration Program: Description. Analysis and Phase I Results," Internal Report. Jet Propulsion Laboratory.

10. J.L. Massey, "Optimum Frame Synchronization,"IEEE Transactions on Communications, Vol. COM-20, No. 2, April 1972.

11. C.N. Georghiades and D.L. Snyder, "Locating Data Frames in Direct-Detection Optjcal Communications," IEEE Transactions on C'ommunications, Vol. COM-32, No. 2. February 1984 .

12. C.N. Georghiades, "Joint Band and Frame Synchronization in Optical Communications," IEEE Transactions on Communications, Vol. COM -33. No. 4, April 1985. 


\section{APPENDIX A}

\section{Derivation of Equation (1)}

For a given alphabet size and sequence length $(N+1)$ let $\operatorname{Pr}\left[K ; j / S_{1}=i\right]$ be the probability that $K$ of the $N$ symbols within the PPM binary subsequence at $j$ are PPM symbols, given that the first symbol in the sequence is $S_{1}=i, i=1.2, \cdots, Q$. For a given $j$, we distinguish two cases: either $i \leq j$ or $i>j$. For $i \leq j$, the probability that $K$ of the $N$ symbols are PPM symbols is the probability that $K$ of these symbols have pulses in the first $j$ slots. Since symbols within a sequence are randomly chosen, the probability that $\hbar^{-}$ of the $N$ symbols have pulses before or at the $j-t h$ slot is $(j / Q)^{K}(1-j / Q)^{N-K}$. Since there are $\left(\begin{array}{c}N \\ K\end{array}\right)$ sets of $K^{-}$symbols we have

$$
\operatorname{Pr}\left[\boldsymbol{K}_{j} ; / S_{1}=i\right]=\left(\begin{array}{l}
N \\
\boldsymbol{K}^{-}
\end{array}\right)(j / Q)^{K}(1-j / Q)^{N-K}, \quad i=1,2, . ., j .
$$

Now. for $Q \geq i>j$, in order for $K$ symbol matches to occur it must be that $\mathrm{K}$ symbols have pulsed slots after the $j-t h$ slot. which implies

$$
\operatorname{Pr}\left[K_{j}^{-} j / S_{1}=i\right]=\left(\begin{array}{l}
N \\
K^{-}
\end{array}\right)(1-j / Q)^{K^{-}}(j / Q)^{N-K^{-}}, \quad i=(j+1), 2 \cdots, Q .
$$

All we need to do now is expectate over all $Q$ first symbols i, all equiprobable. Performing the expectation yields equation (1). 


\section{APPENDIX B}

Derivation of Equations (36) $-(41)$

We first derive equation (36). Here we are interested in the number $D_{0}$ of sequences for which no PPM binary subsequence is a PPM sequence. Our approach is to start with the $Q(N+1)$ possible sequences and then subtract all unwanted sequences. Using proposition 2, we must subtract all sequences that have all their pulses after the $j-t h$ slot for $j=1,2, \cdots,(Q-1)$ and all sequences that have all their pulses before the $j-t h$ slot, $j=1,2, \cdots,(Q-1)$. It is easily seen that there are $(Q-1)^{(N+1)}$ of the former and as many of the letter; $(Q-2)^{(N+1)}$ of these sequences belong to both categories. Subtracting $2(Q-1)^{(N+1)}$ from $Q^{(N-1)}$ and adding $(Q-2)^{(N+1)}$ to avoid subtracting sequences twice yields $(36) .(37)$ is obtained by dividing $(36)$ by $Q^{(N+1)}$.

Let us now compute the number $D_{1}$ of sequences that have exactly one PPM binary subsequence which is a PPM sequence at some shift $j=1.2, \cdots,(Q-1)$. If we let. $\mathrm{X}(\mathrm{j})$ be the number of sequences whose PPM binary subsequence at $j$ is a PPM sequence, we have

$$
D_{1}=\sum_{j=1}^{Q-1} X(j)
$$

For a given $j, X(j)$ is the number of all the sequences that have either all pulses before $j$ or all after $j$. It is easily seen that there are $j^{N+1}$ of the former and $(Q-j)^{N+1}$ of the latter. From the $j^{N+1}$ sequences we must subtract sequences that have all pulses after slot 1 or before slot $(j-1)$ since these are sequences that result in PPM sequence at some slot shift $i<j ;$ there are $2(j-1)^{N+1}-(j-2)^{N+1}$ such sequences for $Q>2$. Similarly, from the $(Q-j)^{N-1}$ sequences we must subtract all those that have either all pulses after the $(j-1)$ slot or all pulses before $(Q-1)($ and after $\mathrm{j})$; there are $\left[2(Q-j-1)^{N+1}-(Q-j-2)^{N+1}\right.$; such sequences. Denoting by $I_{1}(j)$ the number of sequences that have exactly one match at $j$ and have all pulses before $j$ and by $I_{2}(j)$ those that have exactly one match at $j$ and all pulses after $j$. we have for $Q>2$.

$$
I_{1}(j)=\left\{\begin{array}{l}
0, j=1 \\
j^{N+1}-2(j-1)^{N+1}+(j-2)^{N+1}, j=2.3, \cdots(Q-1)
\end{array}\right.
$$


and

$$
I_{2}(j)=\left\{\begin{array}{l}
0,(Q-j)=1 \\
(Q-j)^{N+1}-2(Q-j-1)^{N+1}+(Q-j-2)^{N+1},(Q-j)=2,3, \cdots,(Q-1) .
\end{array}\right.
$$

It is clear that $X(j)=I_{1}(j)+I_{2}(j)$ and that $X(j)=X(Q-j)$. Then

$$
\begin{aligned}
D_{1} & =\sum_{j=1}^{Q-1} X(j)=2 \sum_{j=1}^{Q-1} I_{1}(j) \\
& =2\left[(Q-1)^{N+1}-(Q-2)^{N+1}-1\right], Q>2 .
\end{aligned}
$$

For the special case $Q=2$ it is easily seen by inspection that $D_{1}=2$ which along with (4B) yields (38). Equation (39) is obtained by dividing by $Q^{(N+1)}$.

Finally. to obtain (40) and (41). we observe that in order for all PPM binary subsequences at $j=1.2 \ldots,(Q-1)$ to be valid PPM sequences it must be that all $(N+1)$ svmbols in a sequence are the same. Since there are $Q$ such sequences. we obtain $(40)$ from which (41) follows. 


\section{FIGURE CAPTIONS}

Figure 1: Simulation results for the synchronization probability $P_{c s}$ for $Q=2, N=$ 5,10 .

Figure 2: Simulation results for the synchronization probability $P_{c s}$ for $Q=8, N=$ 20.40 .

Figure 3: Simulation results for the synchronization probability $P_{c s}$ for $Q=16, N=$ 20.40 .

\section{TABLE CAPTIONS}

Table 1: Comparison between simulations and the bound in (42). 
TABLE I. COMPARISON BETWEEN SIMULATIONS

AND THE BOUND IN (42)

\begin{tabular}{|c|c|c|c|c|}
\hline & \multicolumn{2}{|c|}{$\underline{Q=2}$} & \multicolumn{2}{|c|}{$\underline{Q}=4$} \\
\hline$\underline{N}$ & SIMULATIONS & BOUND & SIMULATIONS & BOUND \\
\hline 5 & 0.970 & 0.969 & 0.765 & 0.773 \\
\hline 10 & 0.999 & 0.999 & 0.944 & 0.944 \\
\hline 15 & 1.000 & 1.000 & 0.986 & $0.98 i$ \\
\hline 20 & 1.000 & 1.000 & 0.996 & 0.997 \\
\hline 25 & 1.000 & 1.000 & 0.999 & 0.999 \\
\hline 30 & 1.000 & 1.000 & 1.000 & 1.000 \\
\hline 35 & 1.000 & 1.000 & 1.000 & 1.000 \\
\hline \multirow[t]{2}{*}{40} & 1.000 & 1.000 & 1.000 & 1.000 \\
\hline & \multicolumn{2}{|c|}{$\underline{Q}=\underline{S}$} & \multicolumn{2}{|c|}{$\underline{Q}=16$} \\
\hline$\underline{N}$ & SIMULATIONS & BOUND & SIMULATIONS & $\underline{B O U N D}$ \\
\hline 5 & 0.490 & 0.566 & 0.270 & $0.44 i$ \\
\hline 10 & 0.741 & 0.756 & 0.484 & 0.563 \\
\hline 15 & 0.872 & 0.870 & 0.620 & 0.665 \\
\hline 20 & 0.933 & 0.932 & 0.720 & 0.748 \\
\hline 25 & 0.966 & 0.965 & 0.809 & 0.813 \\
\hline 30 & 0.982 & 0.982 & 0.858 & 0.862 \\
\hline 35 & 0.991 & 0.991 & 0.897 & 0.899 \\
\hline 40 & 0.994 & 0.995 & 0.925 & 0.926 \\
\hline
\end{tabular}




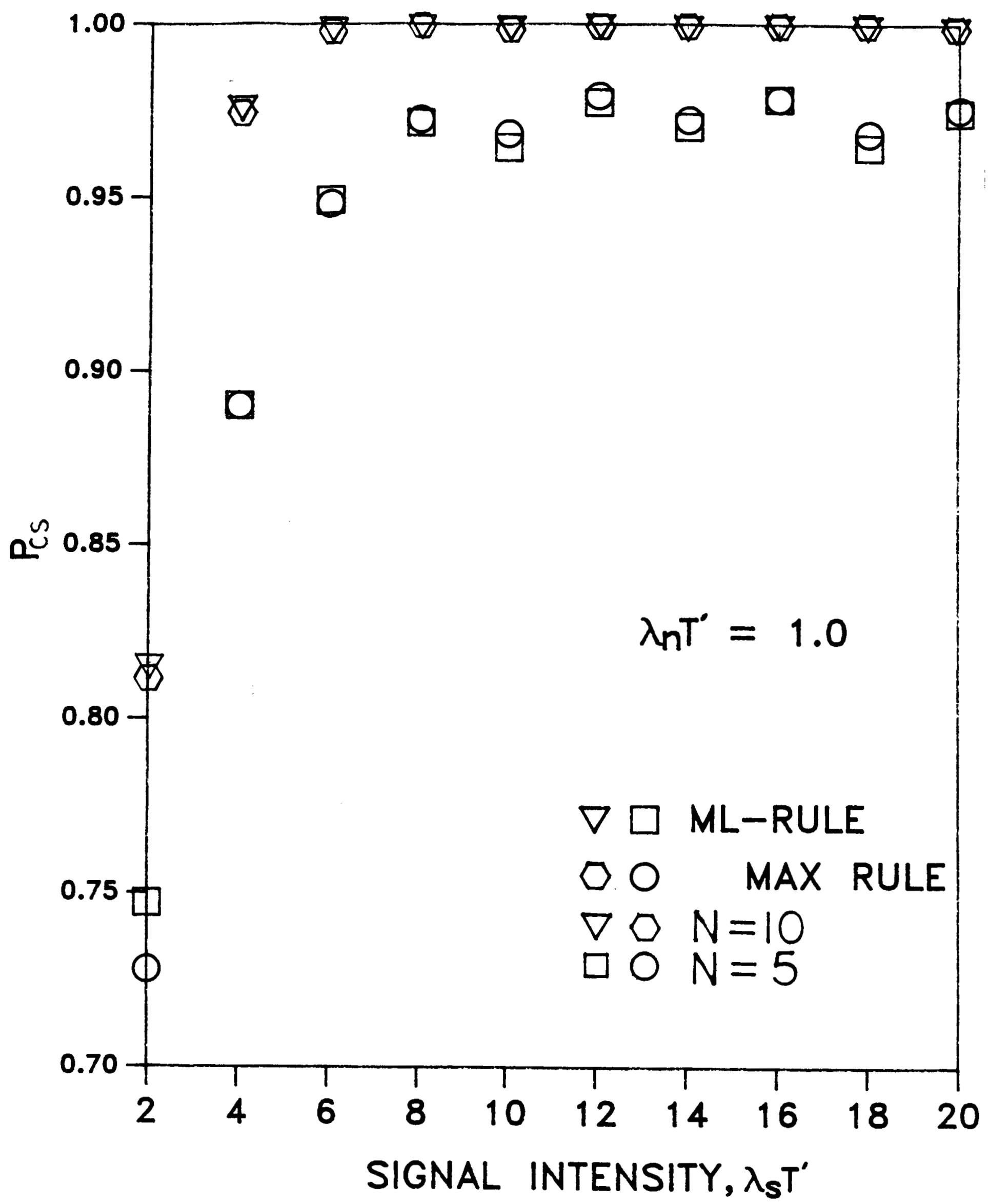

FIG.1 SYNCHRONIZATION PERFORMANCES FOR $(\mathrm{N}, \mathrm{Q})=(5,2),(10,2)$ 


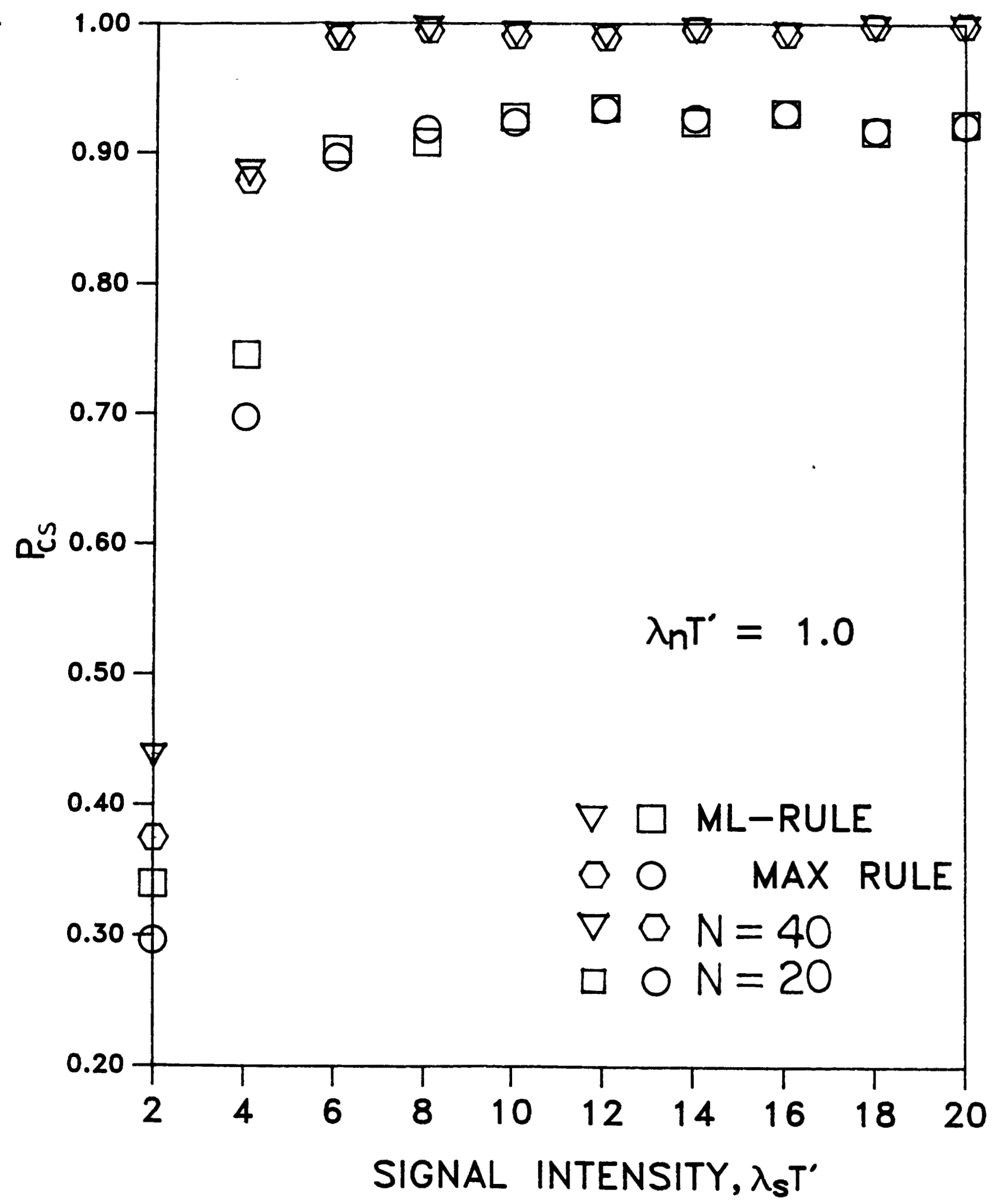

FIG.2 SYNCHRONIZATION PERFORMANCES FOR $(\mathrm{N}, \mathrm{Q})=(20,8),\left(40, \mathrm{Q}^{\circ}\right.$ 


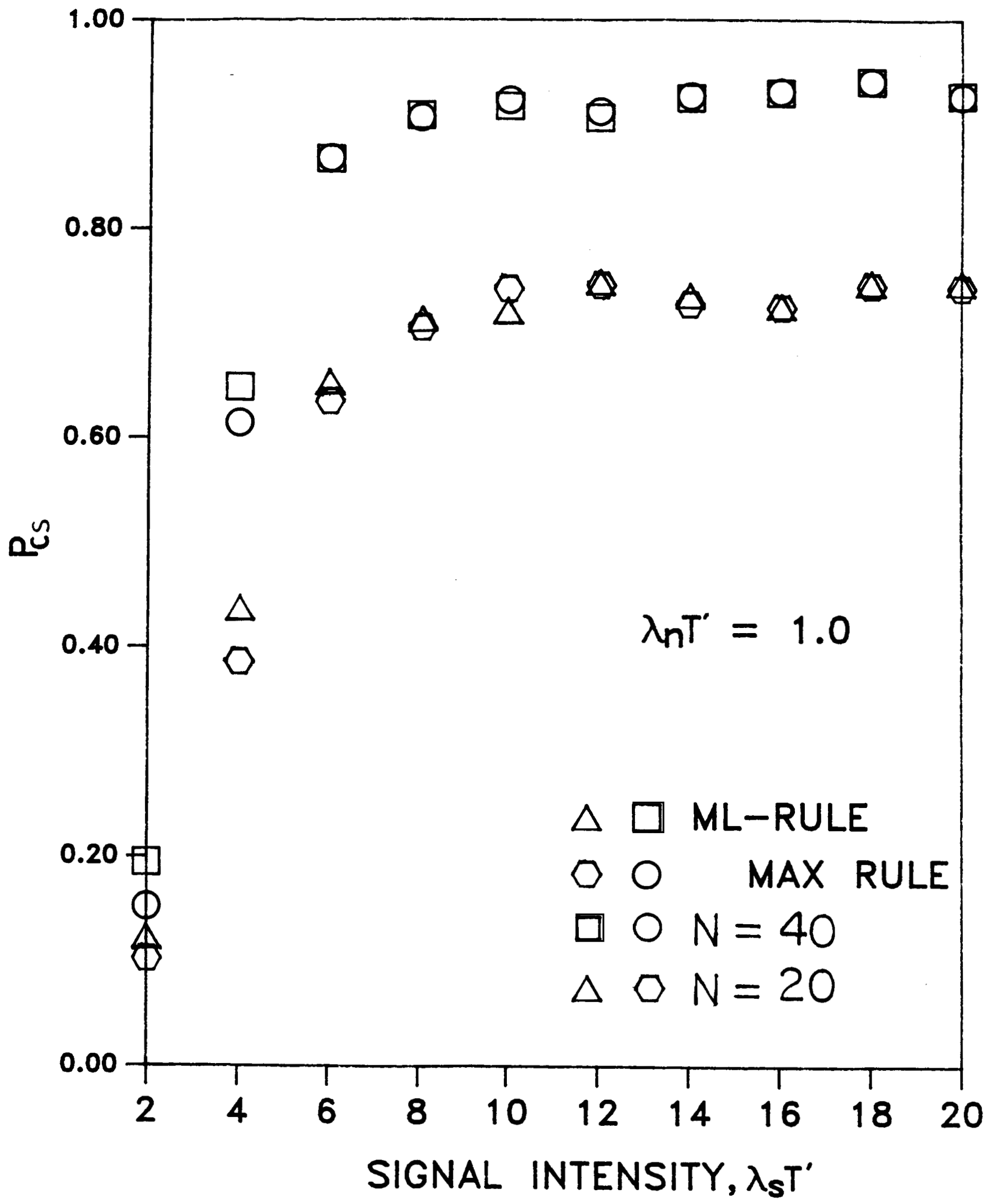

FIG.3 SYNCHRONIZATION PERFORMANCES FOR $(N, Q)=(40,16),(20,16$ 